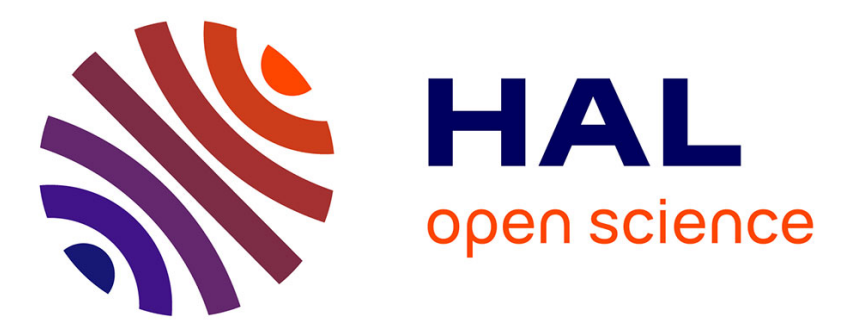

\title{
Efficacité au long terme de la toxine botulique dans l'hyperactivité de vessie chez les patients atteints de sclérose en plaques
}

B. Gabay, D. Maucort-Boulch, A. Ruffion, M.C. Scheiber Nogueira, J.E. Terrier

\section{To cite this version:}

B. Gabay, D. Maucort-Boulch, A. Ruffion, M.C. Scheiber Nogueira, J.E. Terrier. Efficacité au long terme de la toxine botulique dans l'hyperactivité de vessie chez les patients atteints de sclérose en plaques. Progrès en Urologie, 2019, 29, pp.156 - 165. 10.1016/j.purol.2019.02.003 . hal-03486231

\section{HAL Id: hal-03486231 \\ https://hal.science/hal-03486231}

Submitted on 20 Dec 2021

HAL is a multi-disciplinary open access archive for the deposit and dissemination of scientific research documents, whether they are published or not. The documents may come from teaching and research institutions in France or abroad, or from public or private research centers.
L'archive ouverte pluridisciplinaire HAL, est destinée au dépôt et à la diffusion de documents scientifiques de niveau recherche, publiés ou non, émanant des établissements d'enseignement et de recherche français ou étrangers, des laboratoires publics ou privés.

\section{(ㅇ)(1) $\$$}

Distributed under a Creative Commons Attribution - NonCommerciall 4.0 International 
Titre : Efficacité au long terme de la toxine botulique dans l'hyperactivité de vessie chez les patients atteints de sclérose en plaques

\section{Liste des auteurs:}

1. GABAY Benjamin

Service d'urologie, Centre Hospitalier Lyon Sud, 69310 Pierre bénites, Hospices Civils de Lyon, Université Claude-Bernard-Lyon 1-France benjgabay@gmail.com

2. MAUCORT-BOULCH Delphine, Hospices Civils de Lyon, Service de Biostatistique et Bioinformatique, F-69003 Lyon, Université de Lyon, F-69000 Lyon, France Delphine.maucort-boulch@chu-lyon.fr

3. RUFFION Alain,

Service d'urologie, Centre Hospitalier Lyon Sud, 69310 Pierre Bénite, Hospices Civils de Lyon, Université Claude-Bernard-Lyon 1-France;. Alain.ruffion@chu-lyon.fr

4. SCHEIBER NOGUEIRA Maria Carmelita Département de Neurologie Hospices Civils, Lyon, France. mcscheibernogueira@gmail.com 
5. TERRIER Jean Etienne

Service d'urologie, Centre Hospitalier Lyon Sud, 69310 Pierre bénites, Hospices Civils de Lyon, Université Claude-Bernard-Lyon 1-France Jean-etienne.terrier01@chu-lyon.fr 


\section{Efficacité au long terme de la toxine botulique dans}

\section{l'hyperactivité de vessie chez les patients atteints de}

\section{sclérose en plaques}

\section{INTRODUCTION :}

La sclérose en plaques est la première pathologie neuro-dégénérative dans la population jeune(1). La prévalence est de 80/100000 en Europe et elle touche environ 2,3 millions de personnes dans le monde(2)(3). Le diagnostic se fait souvent entre 20 et 40 ans. La symptomatologie urinaire contribue fortement à la morbidité de la pathologie et à l'altération de la qualité de vie(4). La prévalence des signes urinaires est estimée entre $32 \%$ et $96 \%$. L'origine des dysfonctionnements vésicaux est très variable, compte tenu des différents niveaux d'atteinte. On peut observer un syndrome clinique d'urgenturie/pollakiurie pouvant amener à une incontinence urinaire. Ce tableau peut se complexifier avec parfois des manifestations dysuriques amenant à des troubles de la vidange vésicale ou des infections urinaires symptomatiques. Le bilan urodynamique va rechercher une hyperactivité détrusorienne (contractions non inhibées du détrusor, diminution de la capacité vésicale maximale, augmentation des pressions détrusoriennes), mais aussi d'autres anomalies comme une dyssynergie vésico sphinctérienne. A terme ce dysfonctionnement vésical d'origine neurologique lié à la sclérose en plaques, peut avoir des répercussions graves sur le haut appareil urinaire.

Si le diagnostic d'hyperactivité détrusorienne a été porté, il est possible de proposer de première intention des traitements non invasifs comme les anticholinergiques, la neuromodulation tibiale(5)(6). Dans les cas de réponse insuffisante à ces 
traitements, ou d'effets induisant une mauvaise observance du traitement, les options de deuxième ligne doivent être proposées.

La toxine botulique $A(T B)$ en injection intra détrusorienne est un traitement de deuxième ligne dans la prise en charge de l'hyperactivité détrusorienne d'origine neurologique réfractaire aux anticholinergiques(7). L'efficacité à court terme est prouvée(8). L'extension de l'indication chez les patients ne souhaitant pas passer aux auto sondages a été récemment confirmée dans un essai clinique. L'objectif de cette étude était d'analyser les résultats dans la population spécifique dans une population uniquement de patient(s) porteur(ses) de sclérose en plaques plus large que les indications très strictes des protocoles thérapeutiques. L'objectif pragmatique primaire a été d'analyser l'efficacité de la TB dans cette population non sélectionnée. Les objectifs secondaires étaient d'analyser la morbidité et la durée d'efficacité observée en pratique clinique courante.

\section{MATERIELS ET METHODES :}

Notre travail repose sur une étude de cohorte rétrospective, mono centrique.

A partir des dossiers informatisés du service nous avons répertorié l'ensemble des patients majeurs avec un diagnostic avéré de sclérose en plaque, pour lesquels une indication d'injection de toxine botulique intra détrusorienne avait été posée. La période d'étude a été de janvier 2005 à novembre 2015 pour avoir au moins 1 an de recul au moment du travail.

L'indication était systématiquement portée si :

Une hyperactivité détrusorienne était retrouvée au cours du bilan urodynamique (existence de contraction non inhibé du détrusor lors du bilan urodynamique, et/ou existence de fuites urinaires consécutives à une contraction non inhibée au remplissage). 
Le (la) patient(e) était en échec d'un traitement anticholinergique défini, soit par l'absence d'efficacité clinique significative d'au moins deux agents pharmacologiques par voie orale à doses optimales pendant une période d'au moins trois mois chacun, soit par l'interruption du traitement due aux effets indésirables.

La définition de l'efficacité clinique devait être pragmatique dans cette étude rétrospective. Nous avons relevé deux grands critères de succès, parfois mixés. Le critère de succès a été dans un premier temps uniquement basé sur la réduction des épisodes d'impériosité et/ou de fuites urinaire, l'indication primaire étant celle-ci. A la suite de l'étude de Gamé et al (8) nous avons proposé l'injection également à des patients ayant des infections urinaires symptomatiques à répétition et une hyperactivité détrusorienne. Dans ce cas le succès était basé sur la réduction significative (plus de $50 \%$ sur 6 mois) de ces épisodes infectieux aux dires du patient. L'infection urinaire symptomatique était définie par des signes fonctionnels urinaires associés ou non à de la fièvre et confirmée par un examen cytobactériologique des urines (ECBU) positif.

Dans les critères secondaires nous avons relevé la tolérance et les effets secondaires du traitement.

Pour évaluer l'efficacité clinique, nous avons réparti les patients en 4 groupes, en fonction de la fréquence de survenue des fuites et des infections urinaires symptomatiques : efficacité complète, modérée, inefficacité et échec. Cf. Tableau 1 L'effectif des patients analysés à chaque injection, comprenait les patients ayant reçu l'injection, ainsi que les patients en échec de la TB; qui comprenait, les échecs cumulés de l'ensemble des injections précédentes.

Le détail des effectifs analysés à chaque injection était résumé dans le graphique 1 
Les critères de jugement secondaires étaient, dans notre étude : le taux de patients ayant recours au cathétérisme intermittent $(\mathrm{Cl})$ après les injections de TB, l'analyse du taux d'échec de l'injection, son étiologie, la prise en charge alternative proposée. Tous les patients étaient formés aux $\mathrm{Cl}$ avant l'injection. On recommandait aux patients le recours aux $\mathrm{Cl}$ en cas de résidus post mictionnels (RPM) asymptomatiques d'au moins 200 cc, ou bien des RPM inferieurs à 200cc associés à des infections urinaires symptomatiques récidivantes.

L'évaluation des patients intervenait avant la première injection, puis un mois après chaque injection. Un examen clinique complet ainsi qu'un bilan urodynamique étaient faits de façon systématique après les deux premières injections et/ou en cas de réponse intermédiaire. Dans les autre cas, notamment en cas de très bonne réponse clinique le bilan urodynamique n'était pas systématique.

De 2005 à 2009, les patients avaient comme consigne de nous rappeler au moment de la réapparition du critère ayant amené à proposer l'injection. De 2010 à 2015, les patients étaient convoqués de manière systématique à 6 mois puis, en cas de bonne efficacité, le délai de chaque injection était augmenté d'environ 1 mois. En cas de réapparition du critère d'injection, on proposait de rapprocher l'injection, en respectant toujours un délai minimum de 3 mois.

On considérait comme échec du traitement, l'arrêt définitif des injections de TB, toutes causes confondues.

En accord avec notre méthodologiste, les perdus de vue (pdv) étaient censurés si la décision d'arrêt des injections de toxine botulique n'était pas prise à al date des dernières nouvelles. Ils ont été analysés jusqu'au dernières données disponibles. Ils 
n'étaient donc pas systématiquement comptabilisés comme des échecs de traitement par la TB.

L’injection était réalisée soit par le praticien réfèrent soit par un interne.

Les injections se déroulaient majoritairement sous anesthésie locale.

Cette dernière consistait à instiller 30 minutes avant l'injection une solution de deux flacons de LIDOCAINE à $2 \%$ non adrénalinée diluée dans $30 \mathrm{ml}$ de bicarbonate à 14 pour 1000, complétée par l'instillation d'un flacon de LIDOCAINE gel dans l'urètre. Dans de rares cas, une anesthésie générale était nécessaire en fonction du seuil de perception douloureuse de la patiente ou du patient.

Un examen cytobactériologique des urines (ECBU) était réalisé au moins cinq jours avant le geste, et une antibiothérapie était instaurée, si besoin, au moins quarantehuit heures avant la réalisation de l'injection et poursuivie trois jours après.(9)

L’injection se déroulait toujours selon le même protocole. Le patient était installé en position de la taille; nous réalisions une cystoscopie complète, à l'aide d'un cystoscope rigide ou d'un cystofibroscope souple. L'injection intradétrusorienne était réalisée en vingt ou trente sites dispersés de manière homogène dans toute la vessie et à distance du trigone.

Les doses initiales de toxine botulique ont varié au cours du temps, reflétant l'évolution de nos pratiques, et des connaissances.

Avant 2011, les premières injections étaient du DYSPORT 500 Unités DYSPORT (UD) ou 1000 UD, ou du BOTOX 300 Unités ALLERGAN (UA). A partir de 2011, date de l'AMM du BOTOX, les patients ont reçu en priorité du BOTOX 200 UA. Par la suite, du fait de protocoles dédiés à la population SEP qui ne faisait pas de $\mathrm{Cl}$ et des 
travaux concomitants sur les injections dans le cadre de l'HAV idiopathique, les doses de 100 UA ont parfois été utilisées.

Quelle que soit la dose utilisée, les injections étaient répétées au même dosage en cas d'efficacité clinique durable au moins 3 mois. En cas d'inefficacité, ou d'efficacité partielle, on augmentait les dosages lors de l'injection suivante.

Depuis 2011, le protocole du service dans ce cadre est le suivant :

- Passage de 200 à 400 UA de BOTOX dans un premier temps

- Essai de DYSPORT à 1000 UD en cas d'échec.

- Un « rechallenge » est parfois proposé avec le BOTOX 400 UA ou le DYSPORT 1000 UD à la demande du patient avant de considérer que l'on est en échec au traitement.

\section{Analyse statistique:}

Les caractéristiques des patients et des injections étaient résumées par les moyennes (écart-type) pour les variables quantitatives, les effectifs et pourcentages pour les variables qualitatives. L'effet de la première injection sur les infections, et les fuites avait été testé à l'aide d'un test de Mac Nemar.

Les résultats des tests étaient considérés statistiquement significatifs lorsque la valeur de p était inférieure à $5 \%$.La version 3.4.4 de $R$ foundation for statistical computing (C) était utilisée pour les analyses.

\section{RESULTATS:}

De janvier 2005 à novembre 2015119 patients atteints de SEP et souffrant d'une hyperactivité détrusorienne réfractaire aux anticholinergiques ont été sélectionnés pour une première injection intra détrusorienne de TB. La population était majoritairement féminine : 95 femmes $(79,8 \%)$ et 24 hommes $(20,2 \%)$, avec une 
moyenne d'âge de 51,7 ans. Les caractéristiques de la population sont détaillées dans le Tableau 2.

Le suivi médian des patients était de 25,5 mois [1-129]

La majorité des indications $115(96,6 \%)$ avait été posée en raison de plusieurs épisodes quotidiens d'incontinence urinaire. Quatre-vingt-dix-neuf patients (83,2\%) ne présentaient pas d'infection urinaire symptomatique. L'état clinique des patients avant la première injection était détaillé dans le tableau 3.

A la suite de la première injection, la TB intra détrusorienne a été considérée par les cliniciens ayant posé l'indication initiale comme efficace chez 83 patients $(69,7 \%$, cf. tableau 4). Le taux d'efficacité modérée était de 12,6\% (15 patients). La TB était considérée comme inefficace chez 21 patients (17,7\%). Ces patients ont tous été réinjectés à un dosage supérieur après un délai de 3 mois en moyenne.

Au final 4 patients $(3,36 \%)$ n'avaient montré aucune efficacité de la TB malgré l'augmentation des dosages. Le taux d'échec primaire dans notre population était donc de $3,36 \%$.

A la lecture des dossiers, la fréquence des infections urinaires symptomatiques semblaient également diminuée de manière significative, avec 111 (93,3\%) patients sans infections symptomatiques déclarées après la première injection contre 99 $(83,2 \%)$ avant. $p<0,05$ (cf. Tableau 5).

Pour ce qui était de la tolérance, sur les 774 injections réalisées, nous avons retrouvé, une morbidité pour 26 d'entres elles (3,35\%). 10 cas d'asthénie dans la semaine suivant l'injection, 10 injections douloureuses, 1 fausse route urétrale, 2 sepsis urinaires post injection et 3 épisodes d'hématurie post injection, ceux-ci avaient nécessité au maximum une nuit d'hospitalisation pour surveillance. 
En ce qui concerne l'efficacité sur le long terme, Le graphique 2 et 3 montre l'évolution des réponses à la TB intra détrusorienne sur les fuites urinaires et les infections urinaires symptomatiques avec la répétition des injections.

$75,26 \%(70 / 93)$ des patients ne présentaient plus aucune perte urinaire et $80,6 \%(75 / 93)$ des patients ne présentaient pas d'infections urinaires après 3 injections ; $63,23 \%(43 / 68)$ et $72,05 \%(49 / 68)$ à 5 injections ; $53,44 \%(31 / 58)$ et $62,07 \%(36 / 58)$ après 7 injections.

Au delà de la huitième injection, la toxine était efficace pour moins de $50 \%$ des patients dans les deux critères.

La TB était encore efficace chez un patient, après 23 injections.

Le nombre de patients qui utilisaient le $\mathrm{Cl}$ était dans notre analyse de 76 patients $(63,8 \%)$ avant la première injection à 109 patients sur $119(91,6 \%)$ après la première injection. Après 6 injections, 100\% des patients encore traités avaient recours aux $\mathrm{Cl}$. L'évolution du recours au $\mathrm{Cl}$ est détaillée dans le Graphique 4.

Le taux de perdu de vue dans notre étude est de 34/119 patients (29\%). L'évolution de l'effectif des perdus de vue est détaillée dans le graphique 5.

En ce qui concerne l'échec confirmé par le clinicien des injections intra détrusoriennes de TB, notre cohorte a comporté 29 patients $(24,37 \%)$ déclarés en échec de traitement après une durée moyenne de 34,7 mois [1-106].Graphique 6 L'analyse des causes d'échec retrouve une majorité de perte d'efficacité des injections de TB 19 (66\%), une cause liée à l'évolution de la maladie dans près d'un tiers des cas (31\% soit 9 patients) et un cas (3\%) d'un patient ayant souhaité arrêter le traitement sans que la cause puisse être identifiée clairement.

Les traitements alternatifs proposés aux patients après échec sont résumés dans le tableau 6. 
Pour les patients en échec par perte d'efficacité de la TB, et qui étaient encore capable de réaliser des $\mathrm{Cl}$, la technique la plus utilisée a été l'enterocystoplastie d'agrandissement.

Pour les patients dont l'évolution de la maladie empêchait le $\mathrm{Cl}$, la dérivation urinaire trans iléale de type Bricker était la prise en charge la plus proposée.

\section{DISCUSSION :}

L'injection intra détrusorienne de TB est une option qui a été validée dans la prise en charge de l'hyperactivité détrusorienne d'origine neurologique pour des patients blessés médullaires et SEP (9-14).

L'originalité de notre travail est de tenter d'établir, dans une population non sélectionnée, homogène, de patients porteurs de SEP les résultats de ce traitement. II faut rappeler en effet que dans les essais prospectifs qui sont les plus « puissants » sur un plan méthodologique, il existe un certain nombre de biais :

- Sélection d'un «profil » de patients en règle plus sévère que les patients venant consulter (nombre d'épisode quotidien d'incontinence urinaire très élevé dans les essais publiés)

- «Mélange » de patients ayant des profils très différents (spina, SEP, lésion médullaire traumatique)

- Absence de données sur le long terme

Notre étude confirme les excellents résultats d'une première injection de toxine botulique dans la population de patients avec une SEP. Notre taux de bons résultats de $69,7 \%$ se compare favorablement à l'étude de Khan et al (15), avec $72 \%$ de 
patients sec vs $17 \%$ en pré injection. Le taux de $\mathrm{Cl}$ était de $95 \%$ dans cette étude, concordant avec nos résultats puisque nous étions à $91,6 \%$ en post première injection et $100 \%$ après la sixième injection.

La principale alternative à l'injection de toxine botulique est la neurostimulation tibiale (TENS). De seze et al. (16) et al retrouvaient chez 70 patients atteints de SEP, une amélioration clinique dans $82,6 \%$ et $83,3 \%$ des patients à J30 et J90. Rien n'empêche donc de proposer cette alternative avant l'injection de toxine botulique aux patients, surtout si ils ne veulent pas envisager immédiatement l'utilisation de cathétérisme intermittent.

L'efficacité sur le long terme des injections de TB plaide en faveur de cette option si la neuromodulation tibiale et les traitements médicaux n'apportent plus de soulagement aux patients. Giannatoni et al. (17) rapporte des résultats assez stables avec $83 \%$ de patient sans incontinence à 6 ans de suivi. Nos résultats sont moins probants, avec une efficacité supérieure à $50 \%$ seulement jusqu'à la huitième injection (c'est-à-dire autour de la $4^{\text {ème }}$ année de traitement). Cette différence peut s'expliquer en partie par un biais méthodologique puisque nous avons dû analyser les résultats avec un fort taux de perdus de vue. Cette attrition importante de notre cohorte, au détriment des patients les plus « anciens » s'explique en partie par le fait que nous avions été un des premiers services à proposer ce traitement. A l'arrivée de l'AMM en 2011 du BOTOX un certain nombre de patients ont demandé à être traités plus près de leur domicile et ont donc été « censurés » de notre suivi.

Dans la population des SEP, le taux d'infection urinaire symptomatique est particulièrement suivi, du fait des éventuels retentissements sur l'évolution de la maladie et/ou le retard à l'introduction de certains traitements au potentiel immunosuppresseurs. L'étude de Gamé et al.(8) retrouvait une diminution 
significative des infections urinaires symptomatiques après les injections de TB chez des patients atteints de SEP ou de lésions médullaires avec un taux moyen d'infection urinaire qui avait diminué de manière significative, passant de 1,75/6 mois à 0,2/6 mois.

Nous avons également noté une diminution significative des infections urinaires après la première injection. Ce taux faible d'infections urinaires symptomatiques était assez constant avec la répétition des injections puisque nous retrouvions encore 62,07\% d'efficacité complète après sept injections.

II faut cependant insister sur plusieurs biais qui doivent amener à regarder ce dernier résultat avec du recul :

- Le premier biais est inhérent au caractère rétrospectif de notre étude, qui expose à un risque de mauvais recueil de cette donnée, plus difficile à apprécier que la donnée concernant l'incontinence qui était le motif d'injection très majoritaire dans notre population.

- D'autre part, le seul fait de passer au $\mathrm{Cl}$ et donc d'améliorer la vidange vésicale pouvait expliquer la diminution des infections urinaires symptomatiques. Cela constituait un biais certain dans l'analyse du critère. Une analyse en sous groupe en séparant les patients déjà au $\mathrm{Cl}$ en pré injection et ceux sans $\mathrm{Cl}$ permettrait possiblement de s'affranchir de ce biais. Le faible nombre de patients ne permettrait cependant pas de trouver de signification statistique à cette impression subjective. Notre point de vue sur le sujet est cependant assez pragmatique. En effet, de nombreux patients avec une SEP auraient un vrai intérêt à recourir aux $\mathrm{Cl}$. L'entrée dans un chemin clinique d'injection de toxine botulique, avec son risque surajouté de rétention urinaire est parfois un moyen pour les convaincre de l'utilité des $\mathrm{Cl}$. 
En terme de résultats globaux, notre étude semble donc confirmer sur le moyen terme les résultats de la littérature (18-21). En synthèse la toxine botulique intra détrusorienne semble apporter, y compris dans une population non sélectionnée strictement un vrai bénéfice sur la réduction des fuites d'urine et des épisodes d'infection urinaire. Sur le moyen et long terme, cela suppose que les patients acceptent et aient la capacité de faire des $\mathrm{Cl}$ réguliers.

Les faiblesses de notre étude sont nombreuses:

- Le caractère rétrospectif a nécessité une analyse à partir des comptes rendus de consultation. En dehors de protocole, la pratique de questionnaires standardisés (USP, Qualiveen etc.) de bilan urodynamique était trop hétérogène pour permettre une analyse plus poussée de certains éléments des dossiers. Notre critère principal nous semble cependant valide car, comme l'ont démontré Chartier-Kastler et al. (23) les patients traités par des injections répétées de TB pour une hyperactivité de vessie neurologique, recherchent d'abord une disparition des fuites urinaires. Ce critère assez binaire peut assez bien être analysé a posteriori. Dans notre pratique clinique quotidienne, l'utilisation de questionnaire n'a pas d'impact sur la décision clinique et, de ce fait, il est difficile de les imposer. C'est notamment le cas avec les questionnaires dits « de qualité de vie » comme le Qualiveen SF, bien qu'il soit en théorie recommandé(24).

- Bien que le nombre de patient soit significatif, le suivi médian était relativement court, empêchant par exemple l'analyse du taux de réponse en fonction du type d'évolution de SEP, du score EDSS. Nous allons participer à une étude rétrospective nationale recensant le maximum de cas de 
traitements dans cette population spécifique et espérons que l'augmentation du nombre de cas permettra d'avoir des informations sur ce sujet.

- Nous n'avons pas tenu compte des doses injectées et/ou du type de toxine dans l'étude du fait du biais très fort induit par l'évolution des connaissances au cours de la période de l'étude. Les justifications des schémas utilisés ont été décrites dans le chapitre matériel et méthode.

- De même le délai d'injection dans notre étude a été fortement biaisé par la pratique de notre centre, qui a de plus évolué au cours du temps. Notre attitude est le reflet d'une relative hétérogénéité de pratique en France. Initialement nous suivions un premier schéma qui était d'attendre la fin d'efficacité de l'injection avant de proposer une nouvelle injection. La philosophie derrière ce type de prise en charge est de réduire la fréquence d'injection au strict minimum en imaginant que cela pourrait permettre une augmentation de la durée d'efficacité de la toxine. En l'absence de données en rapport avec un intérêt de ce type de schéma, nous avons évolué en 2009 vers le second schéma qui consiste à réinjecter systématiquement les patients après une durée médiane de 6 mois, en augmentant ensuite le délai régulièrement s'ils n'ont pas de symptomatologie gênante avant le jour de l'injection. Ce type de schéma a plusieurs avantages : il permet au patient de ne pas avoir de période d'incontinence. Par ailleurs le service de soin peut plus facilement organiser les injections de la file active des patients.

- Les injections étaient réalisées par différents intervenants dont la qualification variait (internes dès le début de formation, assistants chef de clinique, praticiens). Nous pensons cependant que ce geste est suffisamment 
standardisé pour qu'il ne puisse y avoir qu'un impact très limité sur l'efficacité des injections de toxine chez nos patients.

\section{Conclusion :}

La toxine botulique reste le traitement de référence de l'hyperactivité détrusorienne d'origine neurologique y compris dans la sclérose en plaque. Nous démontrons que dans une population plus large que celle des essais habituellement publiée il existe, au moins à court terme une bonne réponse dans un nombre élevé de cas. Cette réponse peut se maintenir pendant de nombreuses années, notamment si les patients utilisent le cathétérisme intermittent. Nous avons par ailleurs confirmé l'excellente tolérance de ce traitement simple et ambulatoire en pratique courante. Les études rétrospectives du type de celle que nous avons proposé dans cet article ne peuvent cependant apporter que des informations grossières et limitées. La possibilité pour les centres volontaires de participer à des registres prospectifs standardisés aiderait probablement à analyser sur le moyen et très long terme (5 à 10 ans) les évolutions de la sensibilité à ce traitement, afin d'apporter une meilleure information à nos patients. 
1. Compston A, Coles A. Multiple sclerosis. Lancet Lond Engl. 6 avr 2002;359(9313):1221ロ31.

2. Rubin SM. Management of multiple sclerosis: an overview. Dis--Mon DM. juill 2013;59(7):253 $\square 60$.

3. Sand PK, Sand RI. The diagnosis and management of lower urinary tract symptoms in multiple sclerosis patients. Dis--Mon DM. juill 2013;59(7):261ロ8.

4. Zecca C, Riccitelli GC, Disanto G, Singh A, Digesu GA, Panicari L, et al. Urinary incontinence in multiple sclerosis: prevalence, severity and impact on patients' quality of life. Eur J Neurol. juill 2016;23(7):1228 $\square 34$.

5. de Sèze M, Ruffion A, Denys P, Joseph P-A, Perrouin-Verbe B, GENULF. The neurogenic bladder in multiple sclerosis: review of the literature and proposal of management guidelines. Mult Scler Houndmills Basingstoke Engl. août 2007;13(7):915ロ28.

6. Stöhrer M, Blok B, Castro-Diaz D, Chartier-Kastler E, Del Popolo G, Kramer G, et al. EAU guidelines on neurogenic lower urinary tract dysfunction. Eur Urol. juill 2009;56(1):81ロ8.

7. Phé V, Chartier-Kastler E, Panicker JN. Management of neurogenic bladder in patients with multiple sclerosis. Nat Rev Urol. mai 2016;13(5):275ロ88.

8. Gamé X, Castel-Lacanal E, Bentaleb Y, Thiry-Escudié I, De Boissezon X, Malavaud B, et al. Botulinum toxin A detrusor injections in patients with neurogenic detrusor overactivity significantly decrease the incidence of symptomatic urinary tract infections. Eur Urol. mars 2008;53(3):613 $\square 8$

9. Sadiq A, Brucker BM. Management of neurogenic lower urinary tract dysfunction in multiple sclerosis patients. Curr Urol Rep. juill 2015;16(7):44. 
10. Mouttalib S, Khan S, Castel-Lacanal E, Guillotreau J, De Boissezon X, Malavaud B, et al. Risk of urinary tract infection after detrusor botulinum toxin A injections for refractory neurogenic detrusor overactivity in patients with no antibiotic treatment. BJU Int. déc 2010;106(11):1677ロ80.

11. Ginsberg D, Gousse A, Keppenne V, Sievert K-D, Thompson C, Lam W, et al. Phase 3 efficacy and tolerability study of onabotulinumtoxinA for urinary incontinence from neurogenic detrusor overactivity. $J$ Urol. juin 2012;187(6):2131 $\square$.

12. Cruz F, Herschorn S, Aliotta P, Brin M, Thompson C, Lam W, et al. Efficacy and safety of onabotulinumtoxinA in patients with urinary incontinence due to neurogenic detrusor overactivity: a randomised, double-blind, placebo-controlled trial. Eur Urol. oct

13. Kennelly M, Dmochowski R, Schulte-Baukloh H, Ethans K, Del Popolo G, Moore $\mathrm{C}$, et al. Efficacy and safety of onabotulinumtoxinA therapy are sustained over 4 years of treatment in patients with neurogenic detrusor overactivity: Final results of a long-term extension study. Neurourol Urodyn. 24 nov 2015;

14. Rovner E, Dmochowski R, Chapple C, Thompson C, Lam W, Haag-Molkenteller C. OnabotulinumtoxinA improves urodynamic outcomes in patients with neurogenic detrusor overactivity. Neurourol Urodyn. nov 2013;32(8):1109 15 .

15. Khan S, Game X, Kalsi V, Gonzales G, Panicker J, Elneil S, et al. Long-term effect on quality of life of repeat detrusor injections of botulinum neurotoxin-A for detrusor overactivity in patients with multiple sclerosis. J Urol. avr 2011;185(4):1344־9.

16. de Sèze $M$, Raibaut $P$, Gallien $P$, Even-Schneider $A$, Denys $P$, Bonniaud $V$, et al. Transcutaneous posterior tibial nerve stimulation for treatment of the overactive bladder syndrome in multiple sclerosis: Results of a multicenter prospective study. Neurourology and Urodynamics. mars 2011;30(3):306ロ11

17. Giannantoni A, Mearini E, Del Zingaro M, Porena M. Six-year follow-up of botulinum toxin $A$ intradetrusorial injections in patients with refractory neurogenic detrusor overactivity: clinical and urodynamic results. Eur Urol. mars 2009;55(3):705 $\square 11$. 
18. Kalsi V, Gonzales G, Popat R, Apostolidis A, Elneil S, Dasgupta P, et al. Botulinum injections for the treatment of bladder symptoms of multiple sclerosis. Ann Neurol. nov 2007;62(5):452 $\square$.

19. Jabbari B. Botulinum toxin A: a new treatment option for multiple-sclerosisrelated bladder overactivity? Nat Clin Pract Neurol. avr 2008;4(4):188 $\square$.

20. Deffontaines-Rufin S, Weil M, Verollet D, Peyrat L, Amarenco G. Botulinum toxin A for the treatment of neurogenic detrusor overactivity in multiple sclerosis patients. Int Braz J Urol Off J Braz Soc Urol. oct 2011;37(5):642 $\square$.

21. Schulte-Baukloh H, Schobert J, Stolze T, Stürzebecher B, Weiss C, Knispel HH. Efficacy of botulinum-A toxin bladder injections for the treatment of neurogenic detrusor overactivity in multiple sclerosis patients: an objective and subjective analysis.

Neurourol

Urodyn.

2006;25(2):110 $\square$.

22. Mehnert U, Birzele J, Reuter K, Schurch B. The effect of botulinum toxin type a on overactive bladder symptoms in patients with multiple sclerosis: a pilot study. $\mathrm{J}$ Urol. sept 2010;184(3):1011ロ6.

23. Chartier-Kastler E, Rovner E, Hepp Z, Khalaf K, Ni Q, Chancellor M. Patientreported goal achievement following onabotulinumtoxinA treatment in patients with neurogenic detrusor overactivity. Neurourol Urodyn. juin 2016;35(5):595ロ600.

24. Schurch B, Denys P, Kozma CM, Reese PR, Slaton T, Barron R. Reliability and validity of the Incontinence Quality of Life questionnaire in patients with neurogenic urinary incontinence. Arch Phys Med Rehabil. mai 2007;88(5):646 $\square 52$. 
Tableau 1 : Répartition des patients en 4 groupes, en fonction de la fréquence de survenue des événements Table 1: Distribution of patients in $\mathbf{4}$ groups, according to the frequency of occurrence of events

Tableau 2 : Caractéristiques de la population avant la première injection Table 2: Characteristics of the population before the first injection

Tableau 3 : Caractéristiques cliniques de la population avant la première injection de TB

Table 3: Clinical characteristics of the population before the first TB injection

Tableau 4 : efficacité de la TB sur les fuites urinaires après la première injection

Table 4: Efficiency of TB on urinary leakage after the first injection

Tableau 5 : Efficacité de la TB après une injection de TB sur les infections urinaires

Table 5: Efficiency of TB after TB injection on urinary tract infections 
Tableau 6: description des traitements alternatifs des patients en échec de la TB.

Table 6: Description of alternative treatments for patients with TB failure.

Graphique 1 : nombre de patients étudiés à chaque injection Figure 1: number of patients studied at each injection

Graphique 2 : Efficacité de la TB sur les fuites urinaires avec la répétition des injections

Figure 2: Efficiency of TB on urinary leakage with repeated injections

\begin{tabular}{|c|c|}
\hline Echec & Failure \\
\hline Inéfficacité & ineffiency \\
\hline Efficacité modérée & Moderate efficiency \\
\hline Efficacité totale & - Total efficiency \\
\hline
\end{tabular}

Graphique 3 : Efficacité de la TB sur les infections urinaires symptomatiques avec la répétition des injections 
Figure 3: Efficiency of TB on symptomatic urinary tract infections with Repeated injections

\begin{tabular}{|c|c|}
\hline Echec & Failure \\
\hline Inéfficacité & ineffiency \\
\hline Efficacité modérée & Moderate efficiency \\
\hline Efficacité totale & Total efficiency \\
\hline
\end{tabular}

Graphique 4 : Taux de $\mathrm{Cl}$ chez les patients traités par des injections répétés de TB

Figure 4: IC Rates in Patients Treated with Repeated TB Injections
no IC
- Pas de CI
- IC
- CI

Graphique 5: Evolution du nombre de perdue de vue en fonction des injections de TB

Figure 5: Evolution of the number of lost to follow-up according to the injections of TB 
Graphique 6 : Distribution des échecs avec la répétions des injections de TB Figure 6: Distribution of failures with repeat TB injections

Echec
Nombre de patients en cours de traitement

\author{
Failure \\ Number of patients being treated
}




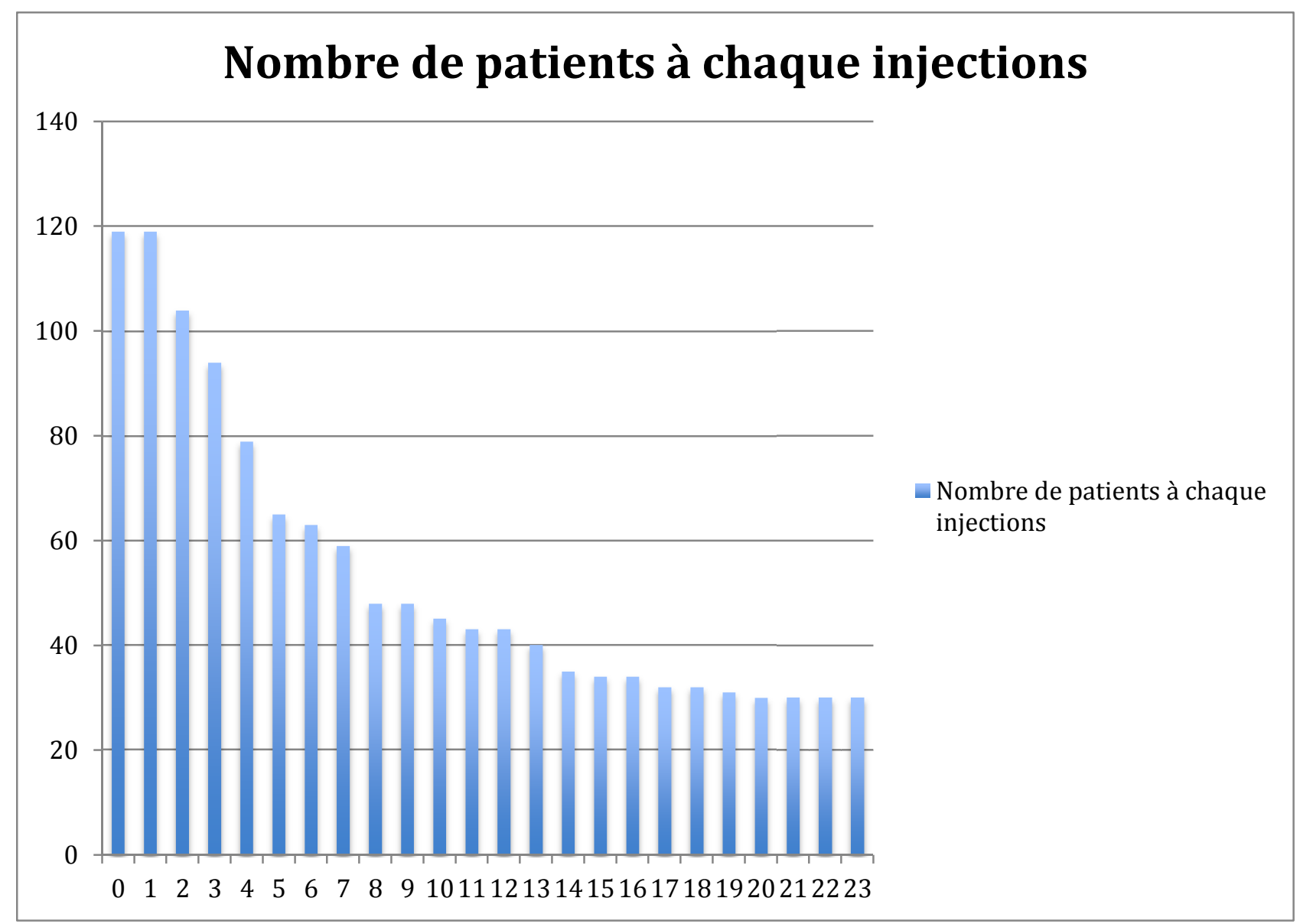

Graphique 1 : nombre de patients étudiés à chaque injection 


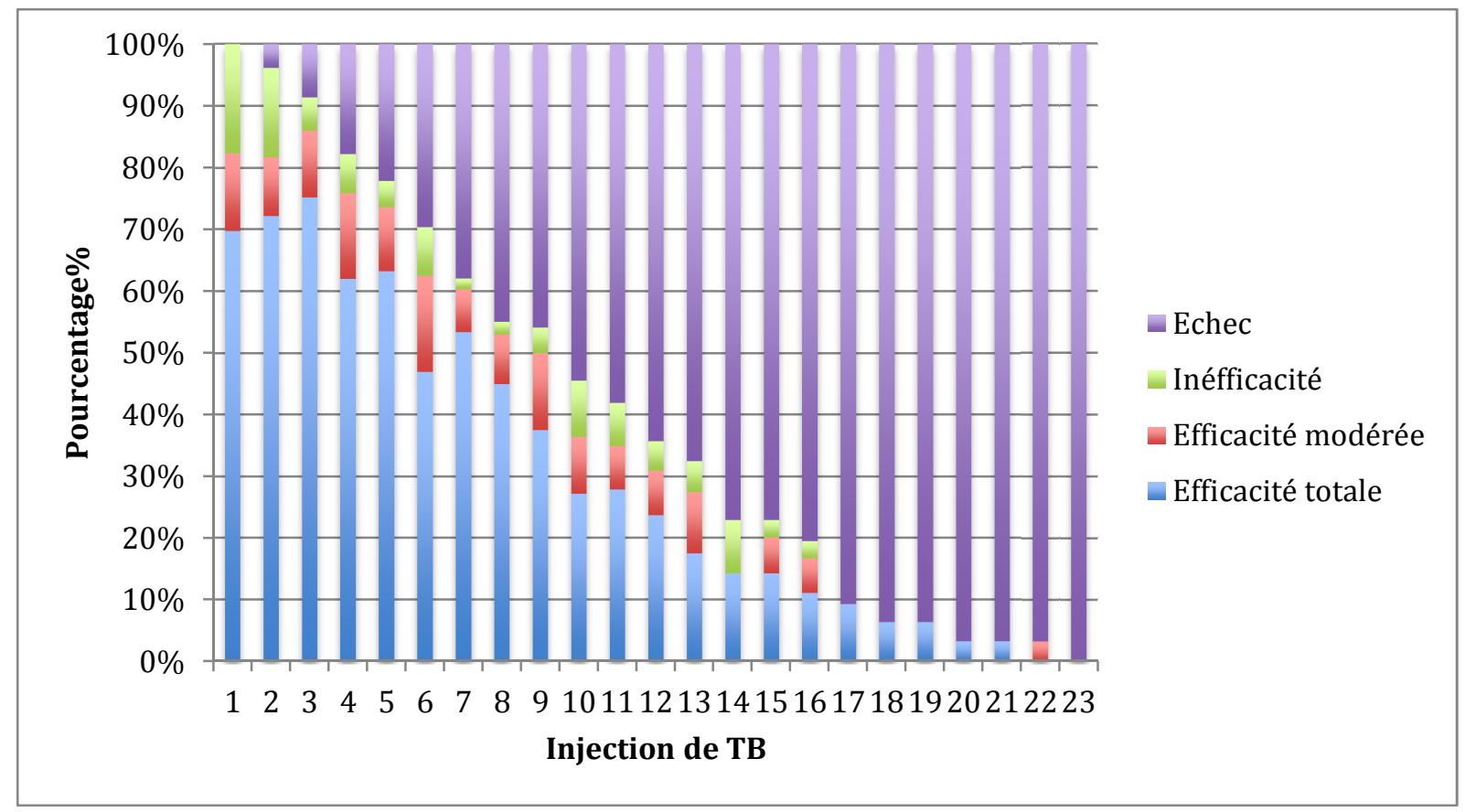

Graphique 2 : Efficacité de la TB sur les fuites urinaires avec la répétition des injections 


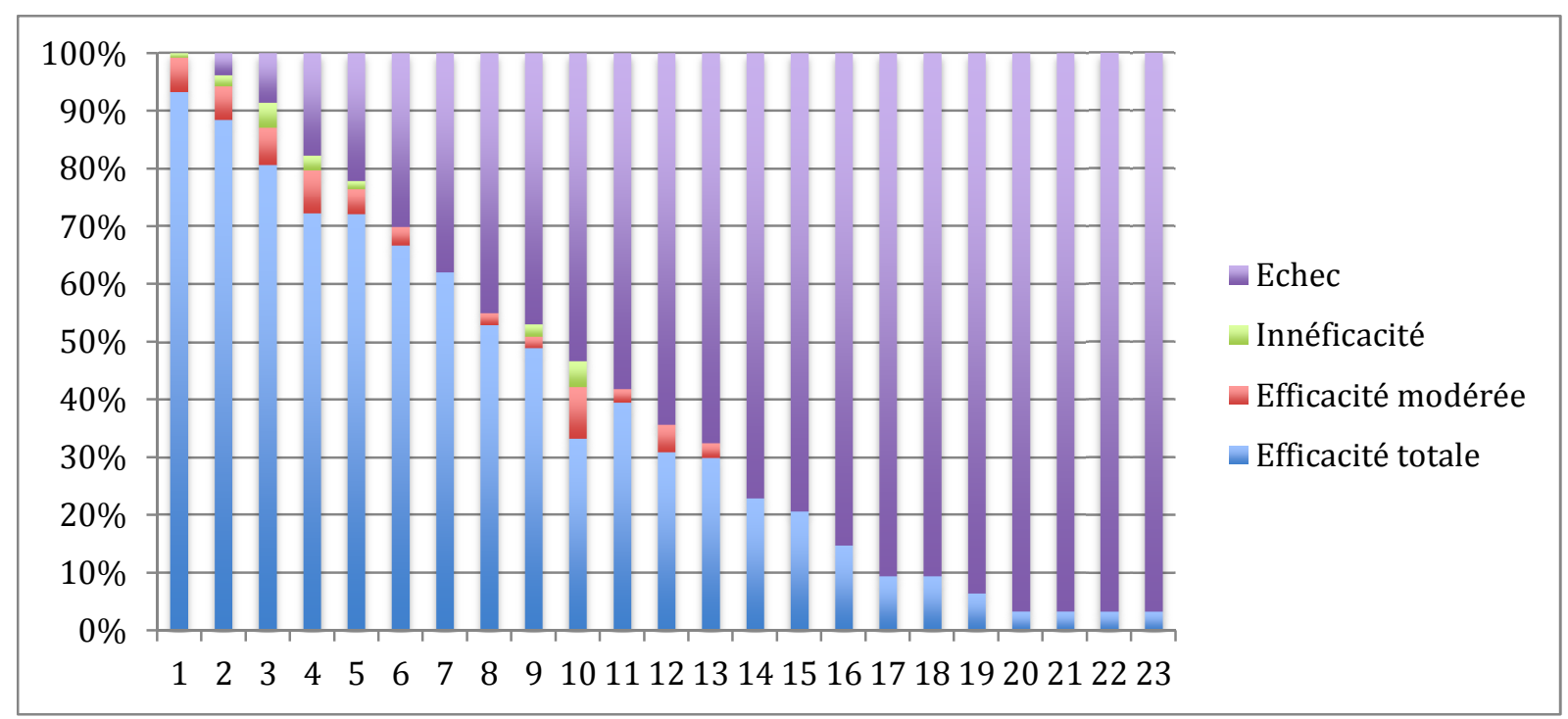

Graphique 3 : Efficacité de la TB sur les infections urinaires symptomatiques avec la répétition des injections 


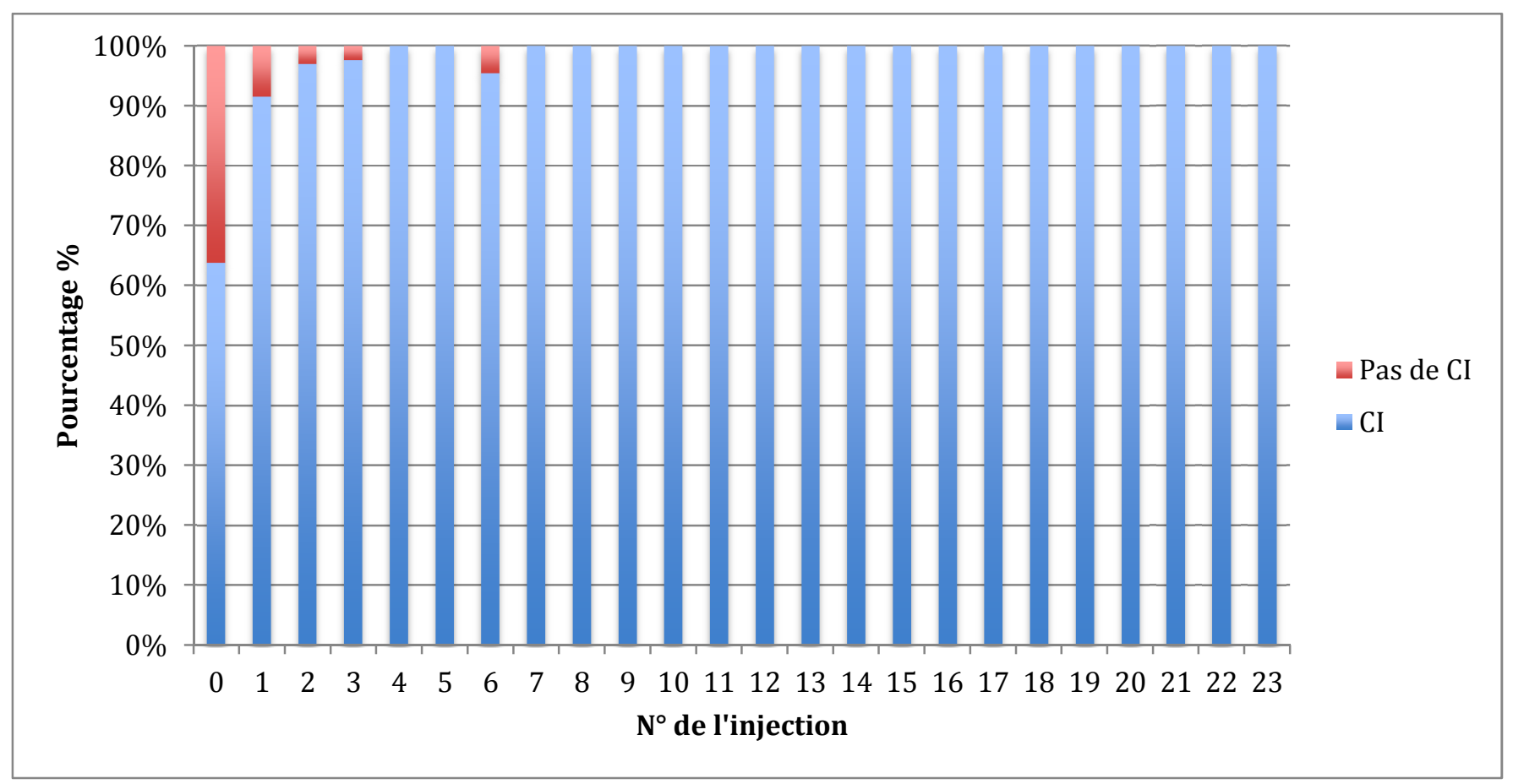

Graphique 3: Taux de CI chez les patients traités par des injections répétés de TB 


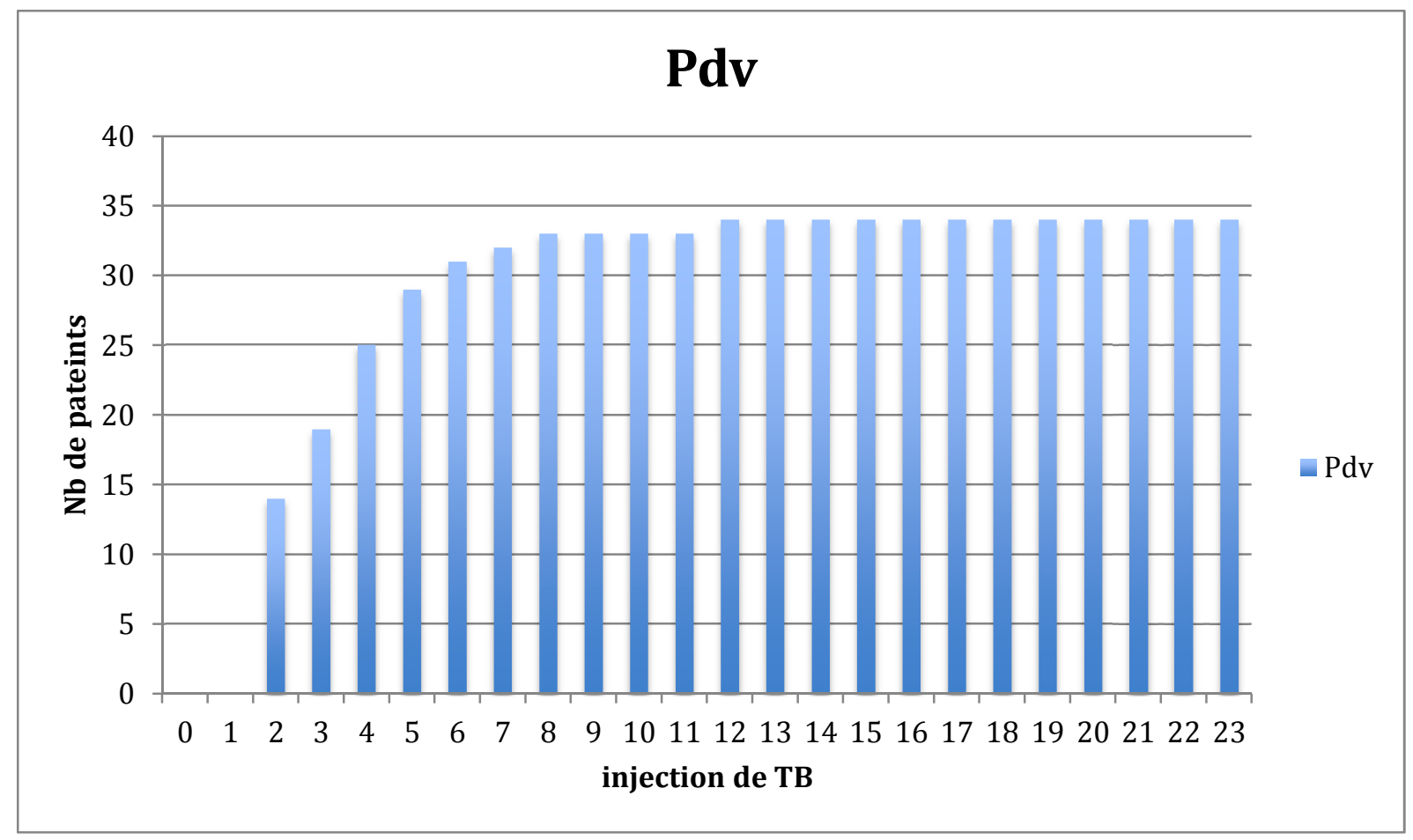

Graphique 5 : Evolution du nombre de perdue de vue en fonction des injections de $\underline{\text { TB }}$ 


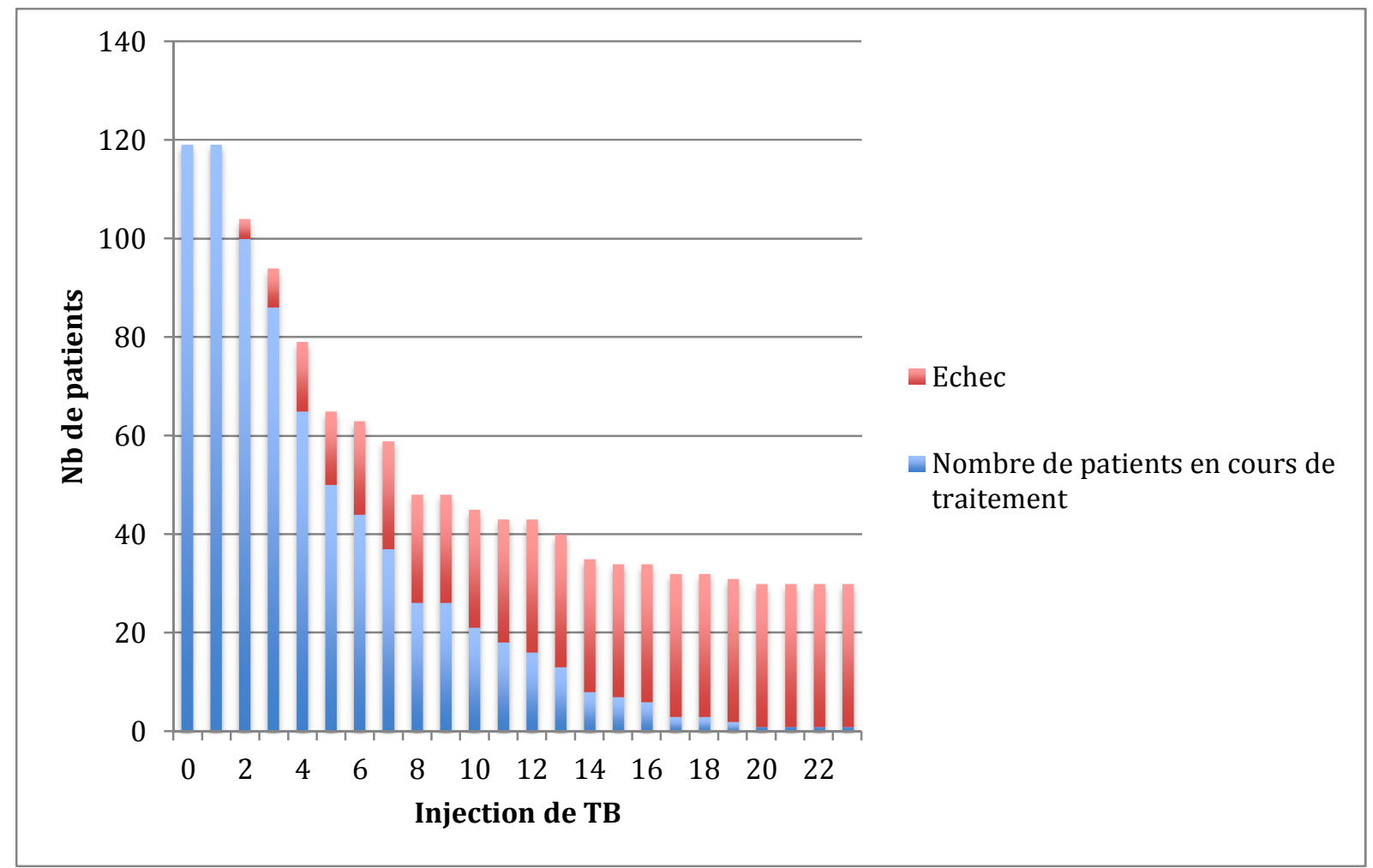

Graphique 6: Distribution des échecs avec la répétions des injections de TB 


\begin{tabular}{|c|c|c|}
\hline & Fuites urinaires & $\begin{array}{l}\text { Infections urinaires } \\
\text { symptomatiques }\end{array}$ \\
\hline Efficacité totale & Absence de fuites & Absence d'infection \\
\hline Efficacité modérée & $<1$ fuite par jour & $\begin{array}{l}<1 \text { infection urinaire par } \\
\text { mois }\end{array}$ \\
\hline Inefficacité & Fuite pluriquotidienne & $\begin{array}{l}\geq 1 \text { infection urinaire par } \\
\text { mois }\end{array}$ \\
\hline Echec & $\begin{array}{l}\text { Patients en échec de la TB } \\
\text { toutes causes confondues }\end{array}$ & $\begin{array}{l}\text { Patients en échec de la TB } \\
\text { toutes causes confondues }\end{array}$ \\
\hline
\end{tabular}

Tableau 1 : Répartition des patients en 4 groupes, en fonction de la fréquence de survenue des événements 


\begin{tabular}{|l|l|}
\hline Âge moyen & $51,7(35-71)$ \\
\hline Sexe: & \\
.Homme nb (\%) & $24 / 119(20,2 \%)$ \\
. Femme nb (\%) & $95 / 119(79,8 \%)$ \\
Ratio Homme/Femme & 0,252 \\
& \\
\hline BOTOX: & $7 / 119(5,88 \%)$ \\
$.100 \mathrm{U}$ nb (\%) & $47 / 119(39,5 \%)$ \\
$.200 \mathrm{U}$ nb (\%) & $45 / 119(37,82 \%)$ \\
$.300 \mathrm{U}$ nb (\%) & $3 / 119(2,52 \%)$ \\
DYSPORT: & $17 / 119(14,28 \%)$ \\
\hline 500U nb (\%) & \\
\hline 1000U nb (\%)
\end{tabular}

Tableau 2 : Caractéristiques de la population avant la première injection 


\begin{tabular}{|l|l|}
\hline Fuites urinaires : & \\
. Quotidiennes nb (\%) & \\
.$\geq 1$ semaine nb (\%) & $115(96,6 \%)$ \\
. Absence de fuite nb (\%) & $1(2,5 \%)$ \\
\hline $\begin{array}{l}\text { Infections urinaires: } \\
. \geq 1 \text { par mois nb (\%) } \\
.<1 \text { par mois nb (\%) } \\
. \text { Absence d'infection }\end{array}$ & $10(8,4 \%)$ \\
& $99(83,2 \%)$ \\
\hline Autosondages & $73 / 119(36,2 \%)$ \\
\hline oui nb (\%) & \\
\hline non nb (\%) & \\
\hline
\end{tabular}

Tableau 3 ; Caractéristiques cliniques de la population avant la première injection $\underline{\text { de TB}}$ 


\begin{tabular}{|c|c|c|c|}
\hline & Fuites Pre 1 & Fuites post 1 & \\
\hline $\begin{array}{l}\text { Fuites } \\
\text { quotidiennes } \\
\mathrm{Nb} \%\end{array}$ & $115(96,6 \%)$ & $21(17,6 \%)$ & \\
\hline $\begin{array}{l}. \geq 1 \text { semaine } \mathrm{nb} \\
(\%) \\
\mathrm{Nb} \%\end{array}$ & $3(2,5 \%)$ & $15(12,6 \%)$ & \\
\hline $\begin{array}{l}\text { Absence de fuites. } \\
\mathrm{Nb} \%\end{array}$ & $1(0,9 \%)$ & $83(69,7 \%)$ & $\mathrm{p}<0,05$ \\
\hline $\begin{array}{l}\text { Totale } \\
\mathrm{Nb} \%\end{array}$ & $119(100 \%)$ & $119(100 \%)$ & \\
\hline
\end{tabular}

Tableau 4 : efficacité de la TB sur les fuites urinaires après la première injection 


\begin{tabular}{|c|c|c|c|}
\hline & Infection pré 1 & Infection post 1 & \\
\hline $\begin{array}{l}. \geq 1 \text { par mois nb } \\
(\%) \\
\mathrm{Nb} \%\end{array}$ & $10(8,4 \%)$ & $1(0,81)$ & \\
\hline $\begin{array}{l}<1 \text { infection } \\
\text { urinaire par mois } \\
\mathrm{Nb} \%\end{array}$ & $10(8,4 \%)$ & $7(5,89 \%)$ & \\
\hline $\begin{array}{l}\text { Absence d'infection } \\
\mathrm{Nb} \%\end{array}$ & $99(83,2 \%)$ & $111(93,3)$ & $\mathrm{p}<0,05$ \\
\hline & $119(100 \%)$ & $119(100 \%)$ & \\
\hline
\end{tabular}

Tableau 5 : Efficacité de la TB après une injection de TB sur les infections urinaires 


\begin{tabular}{|c|c|}
\hline Type d'intervention & $\begin{array}{c}\text { Nb de patients } \\
\mathbf{N b}\end{array}$ \\
$\begin{array}{c}\text { Cystectomies +dérivation } \\
\text { trans-iléale type Bricker }\end{array}$ & 10 \\
\cline { 2 - 3 } $\begin{array}{c}\text { Entérocystoplasties } \\
\text { d'agrandissement }\end{array}$ & 14 \\
\hline $\begin{array}{c}\text { Neuromodulation des } \\
\text { racines sacrées }\end{array}$ & 1 \\
\hline $\begin{array}{c}\text { Cathétérisme vésical au } \\
\text { long court }\end{array}$ & 3 \\
\hline Incontinentation & 1 \\
\hline
\end{tabular}

Tableau 6: description des traitements alternatifs des patients en échec de la TB 